\title{
Plano de amostragem de Acromyrmex spp. (Hymenoptera: Formicidae) em áreas de pré-plantio de Pinus spp.
}

\author{
Acromyrmex spp. (Hymenoptera: Formicidae) sampling plan in Pinus spp. pre-planting areas
}

\author{
Edison Bisognin Cantarelli ${ }^{1}$ Ervandil Corrêa Costa ${ }^{2}$ Ronald Zanettit ${ }^{3}$ Raul Pezzutti $^{4}$
}

\section{RESUMO}

Realizou-se um estudo para elaborar um plano de amostragem de formigas cortadeiras em área de pré-plantio florestal, na empresa Bosques del Plata situada no Departamento de Santo Tomé, província de Corrientes, Argentina. O trabalho teve como objetivo estudar a distribuição espacial de formigueiros do gênero Acromyrmex em área de pré-plantio de Pinus spp., determinar o tamanho ótimo de parcelas e estimar a intensidade amostral desse gênero de formigas. Concluiu-se que a distribuição espacial dos ninhos de Acromyrmex spp. em Corrientes-Argentina, se ajusta ao modelo casual, com o tamanho ótimo de parcela para amostrar a densidade de formigueiros (ninhos. ha $^{-1}$ ) de $700 \mathrm{~m}^{2}$ (10x70m) e a intensidade amostral ótima de $10,5 \%$ da área total para um erro esperado de $24 \%$.

Palavras-chave: reflorestamento; formigas cortadeiras; monitoramento.

\section{ABSTRACT}

A study was conducted to elaborate a sampling plan to leaf cutting ants in forest pre-planting areas, at Bosques del Plata company, located in Santo Tome, Corrientes province, Argentine. The research had as object of study the spatial distribution of Acromyrmex nests in Pinus spp. pre-planting areas to determine the adequate plots size and to estimate the sampling intensity to this genus of ants. It was concluded that the Acromyrmex spp. nests spatial distribution in CorrientesArgentine, adjusts to the randomized model, the adequate plot size to sample the nests density (hole. ha ${ }^{-1}$ ) is $700 \mathrm{~m}^{2}(10 \times 70 \mathrm{~m})$ and the adequate sampling intensity is $10.5 \%$ for a predicted error of $24 \%$.

Key words: reforestation; leaf cutting ants; monitoring.

\section{INTRODUÇÃO}

Algumas empresas do setor florestal brasileiro passaram a adotar sistemas de monitoramento de formigas cortadeiras, com o intuito de aumentar a eficiência e diminuir os custos de combate e, também, reduzir o impacto ambiental decorrente de aplicações sucessivas de inseticidas (ZANETTI et al., 1999).

$O$ sistema de monitoramento adotado na Bahia Sul Celulose S/A, por exemplo permitiu dispensar a operação de combate em $30 \%$ de área monitorada, enquanto nos $70 \%$ restantes foi necessário apenas um combate. Esta estratégia permite um combate a cada 14,3 meses, ou seja, uma redução de $58 \%$ na quantidade de combates, diminuindo os custos e a contaminação, tanto do ambiente quanto dos trabalhadores responsáveis pela operação (MAGESTE \& ANJOS, 1995). O sistema da empresa V\&M Florestal Ltda apresentou resultados semelhantes de redução na área de combate e custos (OLIVEIRA et al., 1993).

A empresa Bosques del Plata está implantando um sistema de monitoramento, o qual trabalha atualmente no plano de amostragem com parcelas circulares de $1000 \mathrm{~m}^{2}$ e uma intensidade amostral de $6,25 \%$, porém não se tem confiabilidade do erro esperado nessa metodologia. Nesse sentido, é importante se fazerem definições corretas e precisas do tamanho da parcela e da intensidade amostral para que não sejam tomadas decisões equivocadas na forma de combate.

\footnotetext{
${ }^{1}$ Departamento de Engenharia Rural, Universidade Federal de Santa Maria (UFSM). Santa Maria, RS, Brasil. E-mail: engedison@yahoo.com

${ }^{2}$ Departamento de Defesa Fitossanitária, UFSM, Campus Universitário, 97105-900, Santa Maria, RS, Brasil. E-mail: eccosta@smail.ufsm.br. Autor para correspondência.

${ }^{3}$ Departamento de Entomologia; Universidade Federal de Lavras (UFLA), Lavras, MG, Brasil. E-mail: zanetti@ufla.br

${ }^{4}$ Empresa Bosques del Plata S.A., Posadas-Misiones, Argentina. E-mail: rpezzutti@cmpc.com.ar
} 
O monitoramento de formigas cortadeiras pode ser executado com vários métodos e a escolha adequada de cada um deles depende, fundamentalmente, de uma definição clara dos seus objetivos, entre os principais estão: indicação do momento ótimo para a intervenção contra as formigas; seleção do método ou métodos a serem adotados; otimização dos recursos em cada método; formação de uma base de dados para geração de uma programação de controle e, por último, avaliação da eficiência da operação (LARANJEIRO, 1994). Os principais métodos de amostragem de formigas cortadeiras incluem a técnica do pior foco, o uso de transectos em linha e em faixas e o uso de parcelas aleatórias. Na primeira técnica, faz-se a avaliação visual da ocorrência de colônias de formigas cortadeiras e de seus danos em área já prémarcada da floresta, procurando caracterizar os focos de forma a perceber aquele em que a situação é mais grave. Por meio de um parâmetro considerado em nível de dano econômico, determina-se ou não a intervenção na área (ANJOS et al., 1993).

O transecto em faixas é uma das formas de amostragem que consiste na contagem do número de formigueiros em faixas de plantio com distâncias variáveis entre elas, começando em uma das bordas do talhão reflorestado e terminando na outra (ZANUNCIO et al., 2002). Normalmente, acompanha as linhas de plantio e limita-se ao espaçamento delas. Já na utilização de parcelas ao acaso, estas são lançadas com área fixa, normalmente retangulares e acompanham a linha de plantio de acordo com um mapa detalhado do talhão, no qual são feitas as escolhas dos pontos, de forma que mostre corretamente toda a área. Existe ainda o transecto em linhas, que consiste no lançamento de linhas de comprimento determinado e largura fixa onde são medidas a distância de cada formigueiro encontrado até a árvore mais próxima e calculada a densidade pelo método Cottan \& Curtis ou Pollard.

O padrão de distribuição espacial dos indivíduos de uma população (ninhos de formigas) é importante no estudo do comportamento e na elaboração de plano de amostragem (CLARK \& EVANS, 1955). ZANETTI et al. (2000) concluíram que as faixas de vegetação nativa reduzem a densidade de sauveiros nos talhões de eucalipto que os margeiam, porém fragmentos florestais aumentam a densidade de sauveiros nos talhões que os margeiam.

A colonização do habitat e a distribuição dos ninhos de Acromyrmex landolti fracticornis, no Paraguai, variam com a declividade, umidade do solo e cobertura vegetal. Além disso, outros fatores, como a alta densidade de outros invertebrados herbívoros, podem limitar a colonização do habitat, após o vôo nupcial das rainhas recém-fecundadas (FOWLER \& ROBINSON, 1977). Essa mesma relação verificou-se no presente estudo no departamento de São Tomé, província de Corrientes-Argentina.

Dessa forma, os objetivos do presente trabalho foram: Estudar a distribuição espacial de formigueiros do gênero Acromyrmex em área de préplantio. Determinar o tamanho ótimo de parcelas para amostragem de formigas cortadeiras em áreas de préplantio de Pinus spp.. Determinar a intensidade amostral que apresenta uma confiabilidade satisfatória.

\section{MATERIAL E MÉTODOS}

Este trabalho foi realizado no campo denominado San Javier, cujas coordenadas UTM (fuso 21) estão entre $6877500 \mathrm{~N}$ e $6867500 \mathrm{~N}$, e entre $6547500 \mathrm{E}$ e 6555000E, da empresa Bosques del Plata S. A., localizada na província de Corrientes-Argentina nos meses de outubro, novembro e dezembro de 2003. Todos os formigueiros visíveis em uma área de 2.750 ha foram localizados, identificados e georreferenciados com um aparelho de GPS (Global Position System), modelo Garmin, antes da implantação da floresta com Pinus sp. no ano de 2004.

Após a coleta dos dados de latitude e longitude de todos os formigueiros, na área de plantio e nas áreas adjacentes, foi gerado um mapa com a localização de cada formigueiro na área. O mapa foi subdividido em amostras de $10 \times 10 \mathrm{~m}$, totalizando 288.547 parcelas de $100 \mathrm{~m}^{2}$.

Foram selecionados dez talhões representativos na área, quanto à densidade de ninhos, totalizando 250 ha, ou 25.106 parcelas. Os dados foram tabulados em planilha eletrônica para análise da distribuição espacial, da determinação do tamanho ótimo das parcelas e da definição da intensidade amostral do monitoramento.

Ressalta-se que, na área de estudo, contabilizaram-se somente os formigueiros do gênero Acromyrmex, que representam $98 \%$ da densidade nas áreas de plantio da empresa Bosques del Plata, pois é realizado um combate diferenciado para esse gênero. As demais espécies de formigas cortadeiras foram desconsideradas, a fim de gerar um plano de monitoramento específico para representar a grande maioria da região.

Determinou-se o tipo de distribuição espacial dos ninhos de Acromyrmex, pelo ajuste do modelo de distribuição de Poisson. Inicialmente foi estimada a freqüência observada dos formigueiros $\left(\mathrm{F}_{\mathrm{obs}}\right)$, pela fórmula: $F_{\text {obs }}=\sum n_{i}$, em que: $\mathrm{n}_{\mathrm{i}}=$ número de formigueiros em cada parcela. 
Em seguida, estimou-se a freqüência esperada $\left(\mathrm{F}_{\text {esp }}\right)$ pelas fórmulas: $F_{\text {esp }}=P(x) \times F_{\text {obs }}$, $P(x)=e^{-\mu}\left(\frac{\mu^{x}}{x !}\right)$,em que: $\mathrm{P}(\mathrm{x})=$ probabilidade de encontrar $\mathrm{x}$ formigueiros; $\mathrm{x}=$ número inteiro $(0,1,2 \ldots) \mathrm{e}$ $\mu=$ média observada de formigueiros (KREBS, 1989).

Obteve-se o padrão de distribuição espacial das formigas cortadeiras, pela comparação entre as freqüências observada e esperada, com a aplicação do teste de Qui-quadrado $\left(\chi^{2}\right)$

$\chi^{2}=\frac{\left(F_{o b s}-F_{\text {esp }}\right)^{2}}{F_{\text {esp }}} \times 100$, testando as hipóteses de que a distribuição é ao acaso ou agregada.

Estimou-se o tamanho ótimo de parcelas pelo método da curvatura máxima de LESSMAN \& ATKINS (1963), inicialmente, as médias e as variâncias da densidade de formigueiros (n.ha $\left.{ }^{-1}\right)$, considerandose o número total de parcelas amostradas de toda a área de estudo.

Posteriormente, dividiu-se o total de parcelas ao meio consecutivamente, até restarem conjuntos de número mínimo de duas parcelas por bloco. A cada divisão, determinaram-se as médias e as variâncias da densidade de formigueiros de cada conjunto de parcelas resultante. Com esses, determinou-se o coeficiente de variação de cada conjunto de parcelas pela fórmula:

$$
\mathrm{CV}=\frac{\sqrt{\mathrm{s}^{2}}}{\overline{\mathrm{x}}}
$$

, em que: $\mathrm{CV}=$ coeficiente de variação; $\mathrm{s}^{2}=$ variância dos conjuntos de parcelas; $\mathrm{x}=$ média dos conjuntos de parcelas.

Em seguida, calculou-se a área (ha) de cada conjunto de parcelas, multiplicando-se o número de parcelas de cada um pela área da parcelas $\left(100 \mathrm{~m}^{2}\right)$. Com esses dados, gerou-se a equação de regressão do coeficiente de variação (CV) em função do tamanho da amostra (ha) para a densidade de formigueiros (n.ha $\left.{ }^{-1}\right)$, por meio do logaritmo neperiano da fórmula:

$$
\mathrm{CV}=\mathrm{a} / \mathrm{y}
$$

, em que: $\mathrm{CV}=$ Coeficiente de variação; $\mathrm{a}$ e b = coeficientes da regressão; $\mathrm{Y}=$ tamanho da amostra.

Com os coeficientes da regressão obtidos, calculou-se tamanho ótimo da parcela pelo método de LESSMAN \& ATKINS (1963), utilizando a expressão apresentada por CHAVES (1985): $\mathrm{N}=\left[\mathrm{a}^{2} \mathrm{~b}^{2}(2 \mathrm{~b}-1) /(\mathrm{b}-\right.$ $2)]^{1 /(2-2 b)}$ em que: $\mathrm{N}=$ tamanho ótimo da parcela; $\mathrm{a}$ e $\mathrm{b}=$ coeficientes da regressão.

Estimou-se a intensidade amostral para a densidade de formigueiros, em níveis percentuais de erro esperado de $1,5,10,15,20,25$ e $30 \%$, dentro de um intervalo de confiança de $95 \%$, pela expressão:

$$
\mathrm{N}=\left(\frac{\mathrm{t}_{(\alpha / 2)} \times \mathrm{s}}{\varepsilon \times \mathrm{X}}\right)^{2}
$$

,em que: $t_{\alpha_{/ 2}}=$ valor tabelado pela distribuição $t$ em função do nível $\alpha$ de $5 \%$ de erro e $(n-1)$ graus de liberdade; $\mathrm{s}=$ desvio padrão dos ninhos do conjunto de parcelas de tamanho ótimo; $\mathrm{x}=$ média da amostra total dos ninhos do conjunto de parcelas de tamanho ótimo; $\varepsilon=$ erro esperado.

\section{RESULTADOS E DISCUSSÃO}

O total de 10.490 formigueiros foram localizados na área de 2.750 ha de pré-plantio de Pinus taeda e Pinus elliottii utilizados neste estudo, sendo 9.521 formigueiros em áreas de plantio e 969 em áreas adjacentes ao plantio (aceiros, estradas, floresta nativa etc.). Obteve-se a média de 3,63 ninhos.ha ${ }^{-1}$. Os dez talhões selecionados representativos, contabilizaram 250,1 ha com um total de 914 formigueiros, ou seja, uma densidade representativa ao censo de 3,62 ninhos.ha ${ }^{-1}$.

Definiu-se a distribuição espacial dos formigueiros do gênero Acromyrmex como casual, pois a freqüência observada foi igual à freqüência esperada, conforme tabela 1.

Colônias de Atta laevigata tendem a distribuir-se uniformemente em locais de alta densidade de sauveiros, apresentando-se ajustadas a um modelo regular de distribuição espacial, mas em áreas menos densas, possuem distribuição casual, como verificado nesse estudo (NICHOLAS \& VILELA, 1995).

No mesmo sentido, em talhões de eucalipto em Minas Gerais, CALDEIRA (2002) concluiu que a distribuição dos sauveiros também foi casual. A distribuição casual ocorre, quando as condições ambientais são semelhantes em qualquer ponto no espaço e a presença de um organismo não interfere na

Tabela 1 - Comparação entre as freqüências observada e esperada, através da aplicação do teste Qui-quadrado. Santa Maria, RS, 2005.

\begin{tabular}{cccc}
\hline$X$ & Fobs & Px & Fesp \\
\hline 0 & 24262 & 0,9642 & 23394,6 \\
1 & 779 & 0,9994 & 778,5 \\
$>2$ & 65 & 1,0000 & 65,0 \\
\hline
\end{tabular}

Qui-quadrado $=0,0000001 \mathrm{p}>0,05$ (não-significativo)

Ciência Rural, v.36, n.2, mar-abr, 2006. 
de outro (BEGON et al., 1996). Isso parece ocorrer em reflorestamentos equiâneos, que se caracterizam pela homogeneidade em tipo de solo, temperatura, tratos culturais etc. Como as iças caem aleatoriamente nessas áreas e recebem combates periódicos do mesmo tipo e intensidade, a tendência é manter essa distribuição casual ao longo do tempo.

Em estudos com colônias de Atta sexdens rubropilosa e Atta laevigata em plantios de Eucalyptus spp., RAMOS et al. (2003) concluíram que a distribuição espacial mostrou-se agregada. Outros trabalhos foram realizados estudando a distribuição espacial de colônias de Atta (FORTI \& PEREIRA-DASILVA, 1979; GOMIDES \& ZANUNCIO, 1995; ZANUNCIO et al., 2002; ZANETTI et al., 2003) e colônias de Acromyrmex (BUCHER \& MONTENEGRO, 1974; FARJI-BRENER, 1995; QUIRAN \& PILATI, 1997; LAPOINTE et al., 1998). Nesses trabalhos fica clara a relação existente entre as espécies de formigas cortadeiras e as características do local de estudo, portanto, os planos de amostragem devem ser regionalizados.

Em estudos com distribuição espacial de Atta sexdens rubropilosa em plantios de Eucalyptus urophylla, ZANUNCIO et al. (2002) concluíram que um bom sistema de monitoramento de ninhos de formigas cortadeiras não deveria considerar apenas o número e a área dos ninhos de formigas, mas também a distribuição espacial em relação à borda de cada talhão, reduzindo assim, a área a ser controlada, o custo da operação e a quantidade de inseticidas no ambiente.

Verificou-se um aumento progressivo dos valores do coeficiente de variação, ao reduzir-se o tamanho da área amostrada, para o parâmetro densidade de formigueiros $\left(\mathrm{n} \mathrm{ha}^{-1}\right)$ (Tabela 2). Isso ocorreu pela redução da área das subparcelas e por um conseqüente aumento do seu número, o que implicou diretamente o acréscimo da variância.

Estimou-se o tamanho ótimo da parcelas de $614 \mathrm{~m}^{2}$ para amostrar a densidade de formigueiros $\left(\mathrm{n} \mathrm{ha}^{-1}\right)$. Como foram utilizadas parcelas de tamanho mínimo de $100 \mathrm{~m}^{2}$, então sugere-se utilizar múltiplos inteiros dessa parcelas, ou seja, retangulares $10 \times 70 \mathrm{~m}$ totalizando uma área de $700 \mathrm{~m}^{2}$.

O cálculo da intensidade amostral do parâmetro densidade de formigueiros $\left(\mathrm{n} \mathrm{ha}^{-1}\right)$ mostrou ser necessário o lançamento de 844,11 parcelas ha ${ }^{-1}$, para $1 \%$ de erro esperado (Tabela 3 ), o que é praticamente inviável economicamente, tendo em vista a intensidade amostral (\%) muito alta. A simulação de um erro esperado de $25 \%$ mostrou ser necessário o lançamento de 1,35 parcelas $\mathrm{ha}^{-1}$ para representar a densidade de formigueiro.
Tabela 2 - Coeficiente de variação da densidade de formigueiros em função do número de subparcelas, obtidas no levantamento em Corrientes, Argentina. Santa Maria, RS, 2005.

\begin{tabular}{lcc}
\hline Número de subparcela & Área da & Coeficiente de \\
& subparcela (ha) & variação (\%) \\
\hline 8.192 & 81,92 & 0,65 \\
4.096 & 40,96 & 0,47 \\
2.048 & 20,48 & 0,41 \\
1.024 & 10,24 & 0,45 \\
512 & 5,12 & 0,49 \\
256 & 2,56 & 0,58 \\
128 & 1,28 & 0,70 \\
64 & 0,64 & 0,89 \\
32 & 0,32 & 1,16 \\
16 & 0,16 & 1,62 \\
8 & 0,08 & 2,23 \\
4 & 0,04 & 3,02 \\
2 & 0,02 & 4,10 \\
\hline
\end{tabular}

Apesar de não se ter conhecimento de estudos, no Brasil, com planos de amostragem para o gênero Acromyrmex, em estudos realizados por OLIVEIRA et al. (1993) com o gênero Atta, utilizaramse parcelas de $720 \mathrm{~m}^{2}$ a cada cinco hectares, para o parâmetro densidade de sauveiros (n.ha $\left.{ }^{-1}\right)$, em áreas de manutenção da empresa V\&M Florestal Ltda, o que representa uma intensidade amostral de $1,44 \%$ da área.

Em plantios de eucalipto em Minas Gerais, o tamanho ótimo de parcela, considerando somente a amostra da densidade de sauveiros, foi de $430 \mathrm{~m}^{2}$, e

Tabela 3 - Número de parcelas de $700 \mathrm{~m}^{2}$ por hectare para amostragem da densidade de formigueiros (n.ha ${ }^{-1}$ ) em função da margem de erro esperada no levantamento realizados em Corrientes, Argentina. Santa Maria, RS, 2005.

\begin{tabular}{lcc}
\hline Erro esperado (\%) & Número de & $\begin{array}{c}\text { Intensidade } \\
\text { amostral (\%) }\end{array}$ \\
\hline 1 & Parcela ha $^{-1}$ & \\
5 & 844,10 & $5.908,78^{*}$ \\
10 & 33,76 & $236,37^{*}$ \\
15 & 8,44 & 59,09 \\
20 & 3,75 & 26,26 \\
24 & 2,11 & 14,77 \\
25 & 1,50 & 10,50 \\
30 & 1,35 & 9,45 \\
\hline
\end{tabular}

* Intensidade amostral superior ao censo total. 
intensidade amostral de 1,72 ou $0,86 \%$ para um erro esperado de 5 ou $10 \%$, respectivamente (CALDEIRA, 2002).

Em estudos de métodos de amostragem para o monitoramento do número e área de colônias de formigas cortadeiras em plantios de Eucalyptus, ZANUNCIO et al. (2004) concluíram que o uso de faixas de $120 \mathrm{~m}$ mostrou-se ser mais precisa do que o uso aleatorizado de parcelas com $720 \mathrm{~m}^{2}$.

A empresa Bosque del Plata trabalha com um custo médio de combate de formigas, no ano de implantação, de $\$ 80,00$ (pesos argentino) por hectare, sendo desses, $\$ 4,75$ o custo médio com o monitoramento. Partindo de que o custo de monitoramento não deva ser superior a $10 \%$ do valor total de combate, sugere-se uma intensidade amostral de $10,5 \%$ para atingir tal definição.

Atualmente a empresa trabalha com parcelas de $1000 \mathrm{~m}^{2}$ e uma intensidade amostral de $6,25 \%$, ou seja, se trabalha com menos intensidade amostral e gastando menos consecutivamente, mas sem conhecer o erro que cometem. Supondo então o uso de 1,5 parcela de $700 \mathrm{~m}^{2}$, temos uma intensidade de $10,5 \%$ o que dará um custo de $7,98 \$ /$ ha, valor próximo a $10 \%$ do custo de combate, representando um erro esperado de $24 \%$.

\section{CONCLUSÕES}

A distribuição espacial dos ninhos de Acromyrmex spp. em Corrientes-Argentina, se ajusta ao modelo casual;

O tamanho ótimo de parcela para a densidade de formigueiros do gênero Acromyrmex ( $\mathrm{n} \mathrm{ha}^{-1}$ ), é de $700 \mathrm{~m}^{2}$ na forma de $10 \times 70 \mathrm{~m}$ e a intensidade amostral ótima das parcelas ao acaso é de $10,5 \%$ para um erro esperado de $24 \%$.

\section{REFERÊNCIAS}

ANJOS, N. et al. Manejo integrado de formigas cortadeiras em reflorestamentos. In: DELLA LUCIA, T.M.C. (ed.). As formigas cortadeiras. Viçosa: UFV, 1993. p.212-241.

BEGON, M. et al. Ecology: individuals, populations and communities. Oxoford: Blackwell Science, 1996. 1068p.

BUCHER, E.H.; MONTENEGRO, R. Hábitos forrajeros de cuatro hormigas simpátridas del gênero Acromyrmex (Hymenoptera: Formicidae). Ecología, v.2, p.47-53, 1974.

CALDEIRA, M.A. Planos de amostragem de sauveiros em eucaliptais. 2002. 39f. Dissertação (Mestrado em Entomologia) - Universidade Federal de Lavras, Lavras.

CHAVES, L.J. Tamanho da parcela para seleção de progênies de milho (Zea mays). 1985. 148f. Tese (Doutorado em Agronomia) - Escola Superior de Agricultura "Luiz de Queiroz", Piracicaba.
CLARK, P.J.; EVANS, F.C. On some aspects of spatial pattern in biological populations. Science, v.121, p.397-398, 1955.

FARJI BRENER, A.G. Ecology of the leaf-cutting ant Acromyrmex lobicornis in Patagonia: Actual distribuition, possible expansion routes and effects on the local plant community. In: INTERNATIONAL PEST ANT SYMPOSIUM, 6., 1995, Ilhéus. Anais... Ilhéus: UESC, 1995. p.101.

FORTI, L.C.; PERREIRA-DA-SILVA, V. Distribuição espacial dos ninhos de Atta spp. (Hymenoptera: Formicidae) em povoamento de Eucalyptus spp. In: JORNADA CIENTÍFICA DA ADC, 8., 1979, Botucatu. Resumos... Botucatu: UNESP, 1979. p.12-22.

FOWLER, H.G.; ROBINSON, S.W. Foraging and grass selection by the grass-cutting ant Acromyrmex landolti fracticornis (Forel) (Hymenoptera, Formicidae) in habitats of introduced forage grasses in Paraguay. Bulletin Entomological Research, v.67, p.659-666, 1977.

GOMIDES, C.H.F.; ZANUNCIO, J.C. Distribuição espacial de ninhos das formigas cortadeiras Atta laevigata (F. Smith) e Atta sexdens rubropilosa Forel: implicações no controle. In: INTERNATIONAL PEST ANT SYMPOSIUM, 6., 1995, Ilhéus. Anais... Ilhéus: UESC, 1995. p.186.

KREBS, C.J. Ecological methodology. Nova Iorque: Harper \& Row, 1989. 654p.

LAPOINTE, S.L. et al. Microgeographic and vertical distribution of Acromyrmex landolti (Hymenoptera: Formicidae) nests in a neotropical savanna. Environment Entomology, v.27, p.636-641, 1998.

LARANJEIRO, A.J. Controle de formigas cortadeiras em reflorestamentos: propagação, operação e monitoramento. In: CURSO DE ATUALIZAÇÃO NO CONTROLE DE FORMIGAS CORTADEIRAS, 2., 1994, Piracicaba. Anais... Piracicaba: IPEF, 1994. 24p.

LESSMAN, K.J.; ARTKINS, R.E. Optimum plot size and relative efficiency of lattice designs for grain sorghum yield tests. Crop Science, Madison, v.3, p.477-481,1963.

MAGESTADE, J.G.; ANJOS, N. Sistema Protatu para monitoramento de formigas cortadeiras. In: CONGRESSO BRASILEIRO DE ENTOMOLOGIA, 15., 1995, Caxambu. Anais... Caxambu: SEB, 1995. p.544.

NICHOLAS, J.T.; VILELA, E.F. Territorial mechanisms in post-nuptial flight gynes of the leaf-cutting ant Atta laevigata (F. Smith). Anais da Sociedade Entomológica do Brasil, v.24, n.2, p.389-400, 1995.

OLIVEIRA, A.C. et al. Um estudo de caso: o sistema de monitoramento e controle de formigas cortadeiras na Mannesmann Fi-EL Florestal Ltda. In: DELLA LUCIA, T.M.C. (Ed.). As formigas cortadeiras. Viçosa-MG, UFV, 1993. p. 242-255.

QUIRAN, E.M.; PILATI, A. Distribution of nests of Acromyrmex lobicornis (Hymenoptera: Formicidae) in a natural semiarid site in La Pampa, Argentina. Revista de la Sociedad Entomológica Argentina, v.56, p.155-157, 1997. 
RAMOS, V.M. et al. Densidade e distribuição espacial de colônias de Atta sexdens rubropilosa e Atta laevigata (Hymenoptera: Formicidae) em área de plantio de Eucalyptus spp. In: SIMPÓSIO DE MIRMECOlOGiA, 16., 2003, Florianópolis. Anais... Florianópolis: CCB/UFSC, 2003. p.174-176.

ZANETTI, et al. Efeito de espécie de eucalipto e da vegetação nativa circundante sobre o custo de combate a sauveiros em eucaliptais. Revista Árvore, Viçosa, v.23, n.3, p.321-325, 1999.

ZANETTI, R. et al. Influência da espécie cultivada e da vegetação nativa circundante na densidade de sauveiros em eucaliptais. Pesquisa Agropecuária Brasileira, Brasília, v.35, n.10, p.1911-1918, 2000.
ZANETTI, R. et al. Distribuição espacial de sauveiros (Hymenoptera: Formicidae) em eucaliptais. In: SIMPÓSIO DE MIRMECOLOGIA, 16., 2003, Florianópolis. Anais... Florianópolis: CCB/UFSC, 2003. p.353-355.

ZANUNCIO, J.C. et al. Spatial distribuition of nests of the leaf-cutting ant Atta sexdens rubropilosa (Hymenoptera: Formicidae) in plantations of Eucalyptus urophylla in Brazil. Sociobiology, v.39, n.2, p.231-242, 2002.

ZANUNCIO, J.C. et al. Sampling methods for monitoring the number and area of colonies of leaf-cutting ants (Hymenoptera: Formicidae) in Eucalyptus plantations in Brazil. Sociobiology, v.44, n.2, p.337-344, 2004. 